\title{
De la mascarade au film vidéo
}

Transmettre le diabolique dans le centre de l'Afrique de l'Ouest

From masquerade to video-film: mediating the demonic in West Africa

\section{Mike Rowlands}

Traducteur : Manuel Benguigui

\section{OpenEdition}

\section{Journals}

Édition électronique

URL : https://journals.openedition.org/tc/6344

DOI $: 10.4000 /$ tc 6344

ISBN : 1952-420X

ISSN : 1952-420X

\section{Éditeur}

Éditions de l'EHESS

Édition imprimée

Date de publication : 15 juin 2012

Pagination : 178-191

ISBN : 2-7351-1512-7

ISSN : 0248-6016

\section{Référence électronique}

Mike Rowlands, « De la mascarade au film vidéo », Techniques \& Culture [En ligne], 58 | 2012, mis en ligne le 30 octobre 2012, consulté le 29 septembre 2022. URL : http://journals.openedition.org/tc/ 6344 ; DOI : https://doi.org/10.4000/tc.6344 


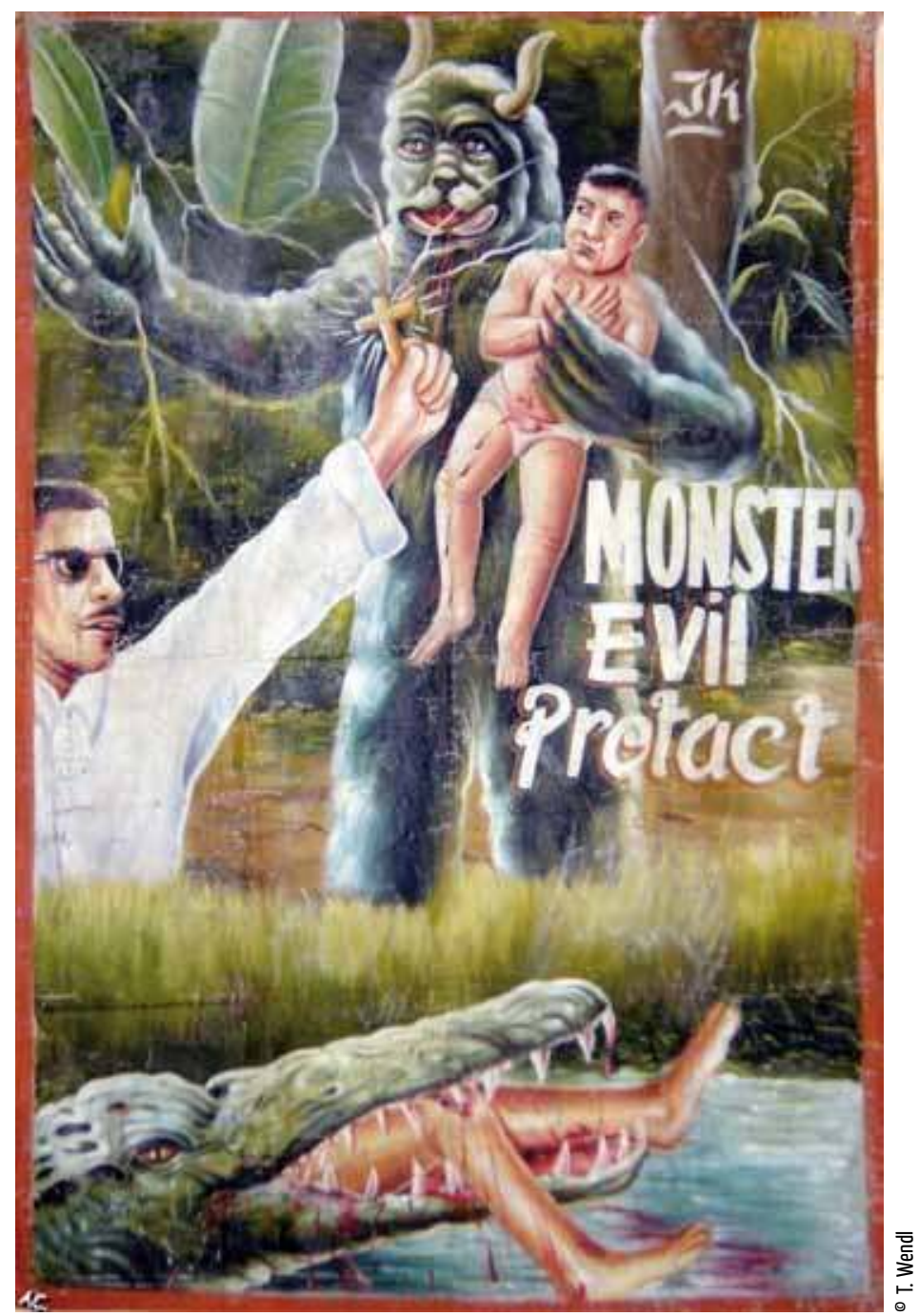




\section{DE LA MASCARADE AU FILM VIDÉO Transmettre le diabolique en Afrique de l'Ouest}

J'ai un problème avec le concept d'objet irremplaçable. Il réside dans l'idée qu'un objet posséderait une essence qui le rendrait irremplaçable. Dire d'un objet qu'il est irremplaçable suppose qu'il soit doté d'un caractère ou d'un contenu particulier comparable à aucun autre. Si une essence existe qui rend un objet irremplaçable, nous devons aussi présupposer un mode singulier de transmission qui conserve le caractère irremplaçable de l'essence contenue dans l'objet. En outre, les objets irremplaçables ne peuvent pas survivre à une exposition frauduleuse ou fausse: tels des reliques, ils ne survivent pas à l'examen conjugué de leur authenticité et de leur efficacité.

Lobjectif du présent article est de démentir le caractère nécessaire de ces présupposés, en termes empiriques, dans le contexte de l'Afrique de l'Ouest. Je compte développer des arguments selon lesquels il est possible, dans le cas qui nous intéresse ici, d'examiner le déplacement de l'idée d'irremplaçabilité d'un objet vers un autre et d'un mode de transmission à un autre. La notion d'irremplaçabilité implique la présence d'une économie du remplaçable, à savoir la recherche de choses nouvelles visant à matérialiser à nouveau l'irremplaçabilité. Si mes arguments ont quelque poids, alors l'idée d'un objet irremplaçable ne peut pas dépendre de ce qu'il représente ou signifie. La singularité ne peut pas être fixée dans la signification mais dépend plutôt de ce qu'est l'objet ou l'image, ou de ce qu'il fait à n'importe quel moment donné. Lanalyse est donc performative plutôt qu'interprétative et si c'est bien Bruno Latour qui est à l'origine du débat, la focalisation sur l'efficacité suggère également que ce qui est considéré comme irremplaçable à un moment donné peut, à un autre moment, devenir non pertinent et remplaçable, perdant ainsi son authenticité et son efficacité. Si l'objet/image ne représente ni ne signifie plus rien mais existe encore, c'est alors par le biais de son potentiel. 


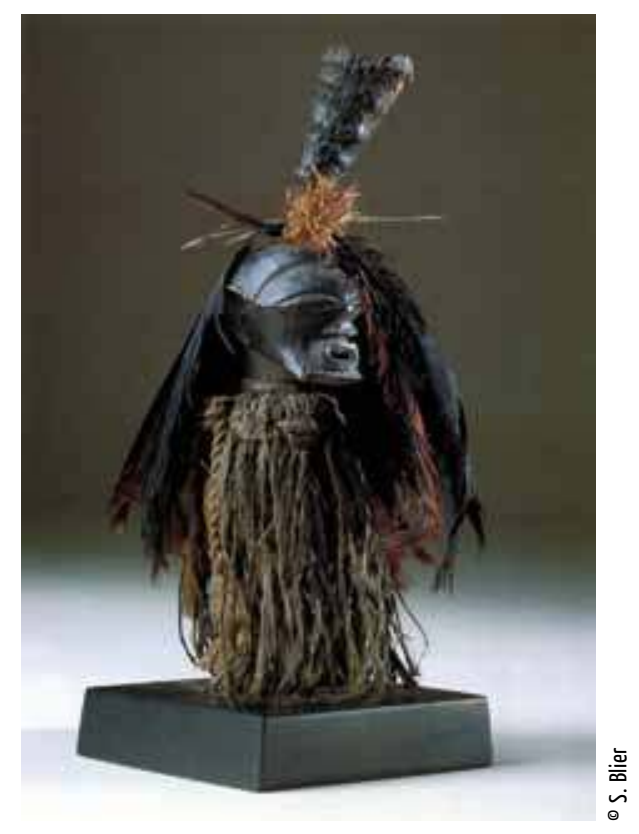

Bo (Fon/Evehe - objet de puissance)

Le concept de fétiche émerge comme résultat de la rencontre des Européens et des Africains lors de la présence portuguaise sur les rivages ouestafricains au xvle et xvile siècles. Fig. 1
La présupposition que je fais à propos de la matérialité des objets irremplaçables reprend deux arguments classiques formulés à propos de la définition des fétiches, en particulier selon la réinterprétation de Marx par William Pietz (1985 : 5-17), Pels (1998) et d'autres:

1. Le fétiche dément une séparation radicale de la matière et de l'esprit ou, comme le souligne Latour, au moins depuis Durkheim, le « prix de l'entrée dans la profession de sociologue » a été de « réaliser que les propriétés propres des objets ne comptent pas, que ces derniers ne sont que de simples réceptacles pour les catégories humaines » (Latour 1993 : 52) - ce qu'on a appelé de façon générale « l'indépendance de l'agentivité de l'objet » (par ex. Gell 1998), bien que les fétiches soient habituellement associés au corps. 2. Comme Pietz l'a noté (1985 : 5), historiquement, « "fétiche" a toujours été un mot de sinistre pedigree ». À l'origine, un produit de la rencontre entre Européens et Africains, en particulier entre les Portugais et les habitants des côtes de l'Afrique de l'Ouest aux XvI e et XvII ${ }^{e}$ siècles, il en est venu à désigner la crainte d'une incapacité à séparer Dieu et le Monde (Graeber 2005).

\section{Des rencontres irremplaçables}

Dans sa Critique du jugement, Kant insiste sur le fait que nous ne pouvons penser la matière vivante comme possible. « Le caractère essentiel de la matière, écrit-il, est l'inertie » (Kant, sect. 73, n. 394). On peut rappeler la longue histoire philosophique de l'Occident, qui oppose les implications d'une perspective kantienne transformant la nature en ressource ou en marchandise, pour souligner à la place la spontanéité de la matière. On pourrait par exemple s'interroger sur ce qui se passerait si l'on prenait sérieusement en compte la vitalité des corps (non humains) (Bennett 2010 : viii). Par ailleurs, comme se le demanderait Latour, que signifierait de traiter une ressource non comme une marchandise, mais comme possédant la vitalité qui n'est pas nécessairement décrite comme étant dotée d'un esprit ou d'une âme? (Latour 2004).

Mais l'insistance sur ce point de la part d'auteurs tels que Gell (1998), Latour (1994) et d'autres, concernant l'efficacité des choses, côtoie difficilement la méfiance européenne vis-à-vis des « faux objets » (les objets qui, tout en étant détachés du corps, peuvent agir sur lui et le contrôler). La crainte des choses qui subvertissent l'être agissant en toute autonomie est historiquement liée à la transformation de la valeur des reliques, qui ont glissé de l'agentivité au signe, au cours de la Contre-Réforme de l'Église catholique en Europe. L'appel protestant à s'opposer à toute sorte de preuve de la présence divine sous la forme d'un esprit ou d'une âme résidant dans des statues/reliques idolâtres au cours de rituels conduits par des prêtres fut contrecarré par la rétention catholique de l'esprit de Dieu devant être rencontré personnellement dans le rituel de la messe. Bien que des centaines de milliers de reliques fussent détruites en Europe entre 1520 et 1650, cela ne signifie pas que la nature irremplaçable des reliques ait été perdue (Figure 2). Dans sa défense contre le protestantisme, le catholicisme se mit à la fois à ressembler à son adversaire et à s'en éloigner. L'Église catholique insista sur le contrôle hiérarchique aux dépens de la croissance incontrôlée des dévotions populaires, en encourageant ce qui 
peut être décrit comme un « programme de modernisation » : l'élimination des « superstitions », qui entraîna en conséquence des évaluations de l'exactitude du type de croyance et de pratique devant être considéré comme superstitieux et de ce qui devait constituer la foi authentique; l'élimination des éléments quasi-commerciaux et des relations d'échange manifestes de la vie de dévotion et l'examen des légendes, des saints, des reliques et des miracles, à la lumière des preuves historiques et critiques. Les reliques en vinrent à être envisagées comme des signes du pouvoir de l'Église, plutôt que comme des éléments dotés de pouvoirs miraculeux. Au cours de la période allant de 1580 à 1730, les reliques des saints qui avaient été récemment redécouvertes dans les catacombes de Rome furent dispersées dans divers endroits du monde catholique, renforçant le pouvoir de l'Église dont Rome était le centre. Mais ces processus apportèrent un nouvel équilibre entre les « vrais et les faux miracles, déplaçant l'attention vers la question de l'authenticité et de la certification, vers la définition des frontières modernes de la foi et de la superstition, de la religion et de la médecine [ou plus généralement de la science] qui est encore en vigueur aujourd'hui » (Gotor 2004 : 123, cité dans Geisbusch 2008). Le fait qu'il soit aujourd'hui possible d'acheter sa relique personnelle sur eBay et de créer son propre autel chez soi (Geisbusch 2008) correspond également à deux autres mouvements de la sacralisation de la collecte d'objets. Le premier, qui coïncide avec le contrôle et l'authentification des reliques, est le fait de collectionner les objets exotiques considérés comme des « curiosités ». À mesure que l'agentivité miraculeuse des reliques s'affaiblit: au moins à partir de 1215 , les reliques doivent être présentées dans des reliquaires fermés, et on ne peut donc les toucher (Concile de Latran IV, canon 62: « que les reliques ne doivent pas être exhibées (ostendantur) en dehors de leur boite »cf. Recht 2008), les collections d'objets des musées apparaissent à leur place et procurent pléthore de merveilles de l'altérité dépouillées de la peur superstitieuse de la capacité de l'objet à soigner ou guérir. L'avènement des musées en Europe, depuis les cabinets de curiosités du XII ${ }^{e}$ siècle jusqu'aux idées de classification de la fin du xviII siècle, a entraîné un second mouvement vers le traitement des objets comme signes. À partir du $\mathrm{xvI}^{\mathrm{e}}$ siècle, au Vatican et dans toute l'Europe, les objets ethnographiques ont été collectionnés parce qu'ils évoquent la capacité à représenter un concept ou une réalité plus large. Ainsi que Peter Pels l'a décrit, les premiers objets de musée sont choisis pour évoquer un sentiment d'émerveillement, l'impression d'être en présence de l'extraordinaire, d'être « au-dehors» ou transporté dans une différence radicale. Et ce sentiment d'émerveillement surgit de sentiments de rupture et de blessure: une interruption dans l'attente des choses du quotidien, causée par la reconnaissance de l'entrée de l'étranger ou de l'étrange dans un monde de routine, de similarité - en somme l'émerveillement face à l'altérité (Pels 1997 : 91-122).

Mais si les objets ethnographiques collectés comme curiosités au xvII siècle partageaient quelque chose avec les reliques (les deux étant caractéristiques du sud de l'Europe catholique), cela avait à voir avec leur matérialité (à savoir que les objets ethnographiques étaient des raretés ou des curiosités qui ne faisaient pas encore partie de collections ordonnées, de taxonomies ou de classifications). Comme les reliques, ils se contentaient d'être: leur

\section{Reliques catholiques}

Alors qu'entre 1520 et 1650 des centaines de milliers de reliques furent détruites en Europe, la nature "irremplaçable" de ces dernières fut conservée. Fermées à clef ou cachées, elles prirent de la valeur dans les collections privées. Fig. 2 


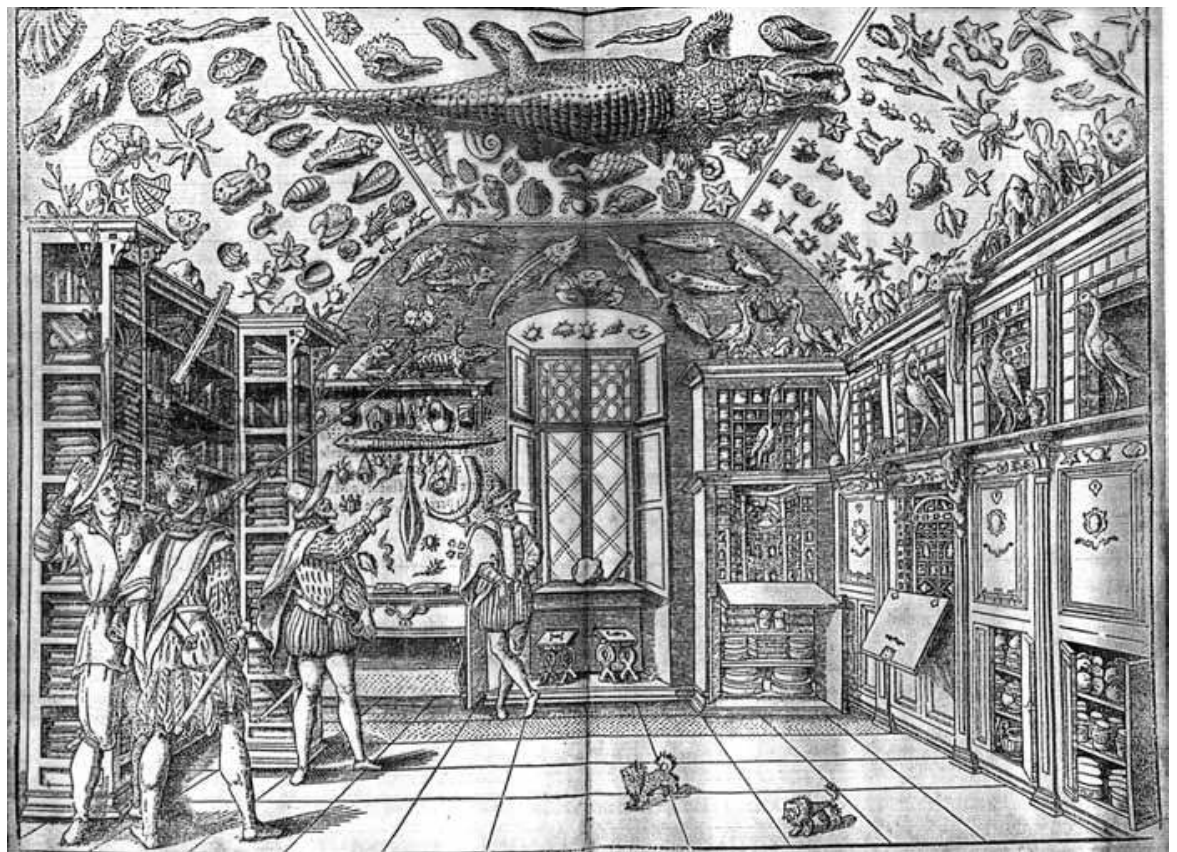

Cabinet de curiosités

Développement des musées en Europe sur le modèle des cabinets de curiosités au xvII siècle jusqu'au classement des objets à la fin du xvIII siècle.

Fig. 3 présence était signe d'émerveillement. Si les reliques reliaient les croyants au monde invisible des saints, la rareté d'un cabinet de curiosités évoquait l'étrange ou l'étranger mystérieux comme un sentiment d'émerveillement, de fascination vis-à-vis de l'altérité. Si les reliques avaient une relation singulière et essentielle avec le corps des saints, le curio ethnographique a intégré, en Europe, un processus plus systématique de documentation et de classification, convertissant les pouvoirs inertes des objets en signes taxonomiques. Mais comme l'a montré Arnold, les cabinets de curiosités du nord de l'Europe en sont venus à être liés à l'expérimentation et à l'alchimie (Arnold 1995). Une autre source d'influence sur le corps a mené in fine au développement de la médecine. Mais tandis que les objets étaient encore des curiosités au XvII ${ }^{e}$ siècle, ils pouvaient être fétichisés, au sens où ils pouvaient être génériques plutôt qu'individuels, magiques plutôt que religieux. De plus, alors que les reliques étaient vidées de leurs associations à des pouvoirs miraculeux, devenant des signes plutôt que des agents, les curiosités ethnographiques acquéraient le pouvoir mystérieux du toucher; elles pouvaient être prises en main et observées, leur pouvoir alchimique exhibé (Classen 2005). Il me semble que c'est quelque part dans la culture historique de cette tendance sécularisatrice à reconnaître certaines qualités « magiques » à des objets et des substances du quotidien que nous pouvons considérer la diffusion de l'idée d'un objet irremplaçable dans la culture populaire européenne. Il est significatif que des choses aussi diverses que des jouets, des maquettes et des portraits acquièrent de la valeur à travers leur irremplaçabilité à la fin du XviII ${ }^{e}$ siècle. La découverte des fétiches lors de la rencontre entre Européens et Africains a constitué une autre caractéristique de l'irremplaçable comme résultat de la relation entremêlée entre reliques et curiosités dans l'Europe catholique du xvII siècle. En conséquence, la crainte des pouvoirs miraculeux des reliques fut exportée vers la périphérie Africaine et prit la forme des fétiches.

\section{Les masques}

Les masques sont indéniablement les objets fétiches correspondant au stéréotype culturel créé dans les relations de l'Europe avec l'Afrique; en témoignent leur nombre dans les musées ainsi que les collections privées et la quantité produite pour le marché touristique. 
Les masques africains étaient déjà considérés comme des icônes essentielles de l'art africain à l'époque où Picasso, Braque et Apollinaire les placèrent à un niveau encore plus élevé de primitivisme dans la création du cubisme. Bataille, reprenant le flambeau de Freud sur la question de l'étrange, envisagea les masques comme un trouble surréaliste permettant l'expression d'une anxiété, un sentiment de danger et un frisson sexuel (Ades 2005). Le caractère unique des masques, qui les rendait authentiques pour le primitivisme, particulièrement le primitivisme africain, relie à son tour l'origine du concept de fétiche aux rencontres entre Européens et Africains. Pietz distingue le fétiche de l'idole par sa «matérialité irréductible » et associe la seconde à une image physique d'une idée ou d'un original immatériel (Pietz 1985). La notion freudienne du fétiche, défini comme un objet qui ne représente rien, renvoie à un sentiment de frisson et d'horreur, tout en sachant qu'en avoir connaissance ne nie pas la tentation d'un désir refoulé (Freud 2002).

Dans l'imaginaire européen, le masque fétiche évoque l'horreur - le monstrueux comme une caractéristique des frontières et des transgressions. Il pousse aussi à une enquête sur la nature de la matérialisation de l'invisible. Lorsque les Européens, au $\mathrm{xvl}^{\mathrm{e}}$ siècle, puisèrent dans le langage du fétichisme (du portugais feiticao) pour décrire le pouvoir des sculptures découvertes sur les côtes de l'Afrique de l'Ouest comme objets capables d'agentivité physique, la réaction fut façonnée, à l'époque, par la redéfinition de la signification du rejet des reliques par la Contre-Réforme catholique. Lors de la rencontre entre Européens et Africains de l'Ouest, l'extériorité du pouvoir superstitieux de la relique à la découverte de l'objet ethnographique comme fétiche, constitua un élément important. La description de masques et de figures africains comme fétiches débute presque au moment des premiers contacts avec les Portugais au Xvi siècle (Pietz 1985 : 7). Le fétichisme était attribué aux Africains de l'Ouest comme un signe de leur incapacité à appréhender la présence (invisible) du monde des esprits. La distinction catholique entre sorcellerie et religion devint superflue : la magie et la religion ritualisée étaient une seule et même chose. Si le protestantisme avait assuré qu'il ne pouvait y avoir de contact matériel avec un Dieu transcendant - la médiation n'étant possible qu'à travers la prière et la lecture de textes sacrés -, et que, de son côté, le catholicisme s'était accroché à une signification littérale de la messe tout en rejetant la matérialité des objets comme moyen de rencontre directe avec le sacré, dans la périphérie de l'Afrique de l'Ouest, une troisième possibilité d'une relation animiste avec le monde invisible des esprits fut mise au jour à travers le toucher des sculptures et des masques. Cette troisième relation tactile se compléta de l'importation de tissus européens, d'objets en métal, de perles, de céramiques, et de bouteilles contenant (ou pas) de l'alcool, tous éléments qui furent rapidement assimilés comme moyens de renforcement de la médiation. Lorsque les Portugais disparurent de la scène, les voyageurs protestants qui vinrent à leur suite reprirent l'idée générale que les fétiches n'étaient pas simplement des charmes magiques: c'étaient les dieux des Africains et le produit de «faux prêtres » et de l'ignorance.

Étant donné leur forme vaguement anthropomorphe, il n'est peut-être pas surprenant que les masques aient été considérés comme prééminents dans la caractérisation des fétiches religieux, au sens où ils étaient des objets matériels dotés d'un certain animisme. Selon Philippe Descola, les masques exhibaient cependant une valeur chimérique comme exemples de son monde de l'enchevêtrement, où les images peuvent posséder une valeur additionnelle lorsqu'ils sont composés d'éléments d'origines diverses (Descola 2010). Contrairement à l'expérience chrétienne de la relique comme signe de béatification, le fétiche africain est devenu son opposé: un signe de médiation matérielle vers un monde 


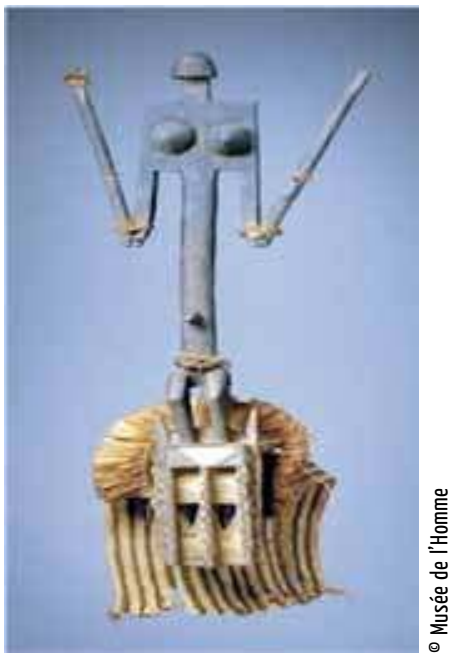

Masque Dogon provenant de la mission Griaule

Les masques africains furent immédiatement sélectionnés comme des icones majeures de l'art africain à l'époque où Picasso, Braque et Apollinaire les élevaient à un haut degré de primitivité consubstantif du moment Cubiste.

Fig. 4 monstrueux de sorcellerie/maléfice et d'horreur. Le point essentiel est que, pour la relique au sens chrétien médiéval, le lien direct entre le monde invisible et l'objet réside dans l'incarnation physique du Christ et des apôtres, et dans les béatifications subséquentes des saints et des qualités métonymiques de toucher, de prise en main et de contact de leurs substances corporelles. Ce qui a accompagné l'interprétation européenne du masque africain comme fétiche, c'est la pensée horrible qu'un esprit extérieur pouvait littéralement envahir l'objet ou le corps. Le fétiche était l'inverse de la relique, et on considérait qu'entrer en contact avec le fétiche corrompait la chair et endommageait l'âme du Chrétien converti. D’où la longue hostilité envers les « objets paiens » entretenue par les missionnaires portugais, néerlandais ou espagnols sur les côtes de l'Afrique de l'Ouest au cours des XVI ${ }^{e}$ et xVIII ${ }^{e}$ siècles. Jusqu'à la fin du xviIIe siècle, les fétiches étaient brûlés ou détruits par les Européens, jusqu'au moment où les principes de la science et de la classification évolutionniste firent de leur collecte un mode plus responsable de conservation. En fait, les métaphores européennes d'invasion corporelle n'étaient pas appropriées à la compréhension des concepts indigènes de substances corporelles et de leur transmission dans une bonne partie de l'Afrique de l'Ouest et l'Afrique Centrale, mais les critiques fondées sur une compréhension ethnographique plus substantielle des réalités africaines ne se seraient pas révélées pertinentes dans ce cadre. Au lieu de quoi, c'est précisément la nature hybride de la façon dont les idées de la relique débarrassée de ses qualités animistes mais conservant son image de pouvoir quant à la guérison des corps et des âmes qui entra dans les idées chrétiennes de conversion et d'appartenance à la fois des Européens et des Africains.

\section{Rencontres « tribales »}

Sur une période longue de plusieurs siècles, en particulier au cours des contacts avec les missionnaires, il n'est pas plausible d'imaginer une structure duelle dans laquelle la crainte européenne des masques comme fétiches matérialisés et une esthétique africaine indépendante se seraient maintenues indépendamment l'une de l'autre. C'est incompatible avec l'idée de masque. Sur de nombreux points, les masques sont une conséquence de la rencontre entre Européens et Africains. Différentes formes peuvent être identifiées.

Premièrement, les masques comme production hybride de cette rencontre apparaissent d'abord comme des produits de collections muséales décontextualisées ( $c f$. Figure 4 masque de Michel Leiris collecté lors de l'expédition Dakar-Djibouti). Les anthropologues spécialisés dans l'art africain, par exemple, ont passé les quarante dernières années à établir que l'image d'art tribal des masques africains n'avait pas de lien avec l'étude des mascarades, à savoir lorsque les masques font partie d'une représentation incluant des danses, de la musique et des corps humains matériellement transformés. On ne peut pas non plus modifier la signification d'un masque isolé en le recontextualisant. Les masques, ou pour le coup les figures fétiches de manière plus générale, sont montrés comme possédant une présence matérielle et spirituelle prouvant qu'ils sont capables d'avoir un pouvoir en eux-mêmes.

Deuxièmement, il existe des exemples pour lesquels la rencontre avec le primitivisme européen a transformé l'image en tant qu'icône. En 1958, l'artiste autrichienne Susanne 
Wenger, qui habitait au Nigéria, fut approchée par une délégation d'officiels de la divinité Yoruba Osun qui lui demanda son aide pour restaurer les autels de la ville d'Osogbo. Ces autels s'abîmaient depuis plusieurs années, et la forêt sacrée était envahie par les agriculteurs et les hommes d'affaires (Probst 2007). Fascinée par la divinité, Wenger accepta la demande et les restaurations commencèrent avec l'autel de Idi Baba, situé aux abords de Osogbo, sur la route de Ibokun (Figure 5). Elle travailla ensuite sur le temple principal d'Osun situé dans la forêt. Cependant, ce qui devait initialement constituer un ravalement de façade mineur finit par aboutir à une débauche d'icones. Des petites statues jusqu'aux autels les plus imposants, la forêt finit par être inondée de nouvelles représentations picturales. Du point de vue conceptuel, en prêtant aux diverses divinités Yoruba censées habiter la forêt une nouvelle présence matérielle, l'idée était de réunir l'art et la culture, de façon à combler l'absence que les effets aliénants du colonialisme et du capitalisme avaient fait subir à la société Yoruba. En d'autres termes, et formulé à la façon de Weber, il s'est opéré un glissement des processus coloniaux de désenchantement vers un projet colonial de réenchantement (Probst 2007 : 103). Dans le cas de Wenger, l'expérience a consisté en une création délibérée de nouvelles images iconiques « exprimant la phase fluide, ouverte et encore indéterminée que traversait, pensaiton alors, la société Yoruba » (Probst 2007 : 103; Ogundele 2003 : 107). En 1965, cinq ans après l'Indépendance, le gouvernement nigérian déclara la forêt d'Osun monument national. Dans les années 1970, le festival d'Osun fut mis en place et devint dans les deux décennies suivantes l'un des plus grands festivals culturels du Nigéria ainsi qu'un site important de mémoire pour la diaspora. Le site est classé au Patrimoine culturel de l'Unesco depuis juillet 2005 (Probst 2007 : 104).

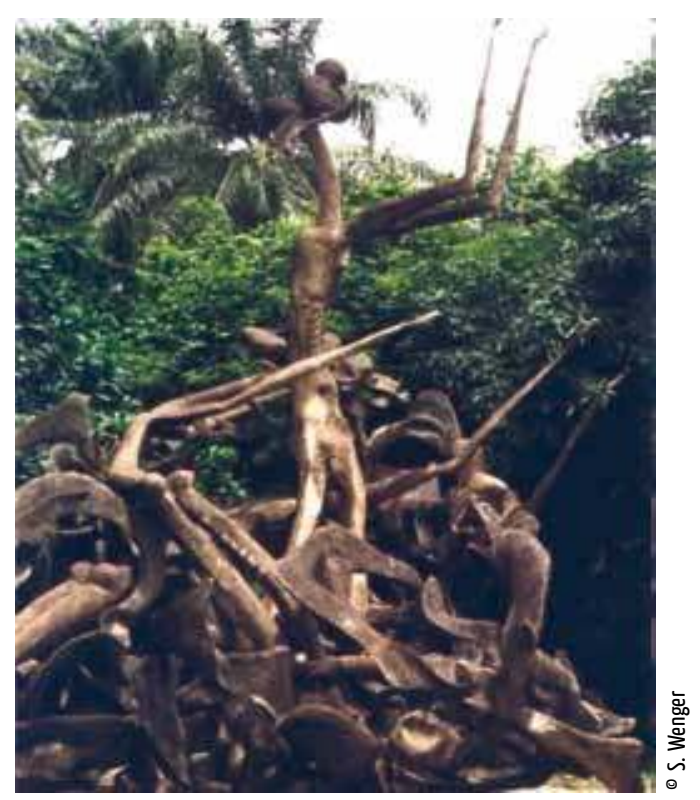

\section{Autel, Oshogbo}

La maison autel Oshogbo de Susanne Wenger comme bataille iconique. S'échelonnant de petites et modestes effigies à des autels imposants, le bois sacré fut peuplé de nouveaux apports figuratifs.

Fig. 5

\section{Lier les mots aux objets}

La relation entre les mots et les objets est centrale pour la compréhension d'un autre aspect de la rencontre entre Européens et Africains, à savoir l'impact du christianisme missionnaire. Le statut du langage a été crucial dans les origines de la tradition protestante de la Réforme en Europe, puisque la prière et la lecture à voix haute remplacèrent la tradition catholique du rituel et de la vénération des objets et des images. Comme l'a montré Talal Asad, la sincérité du croyant chrétien est fondée sur la croyance selon laquelle les mots donnent une expression extérieure aux intentions intérieures des personnes. Le langage constitue le médiateur fondamental entre le sujet et l'objet, et la meilleure façon d'évaluer la pureté du discours du croyant réside dans son intention (Asad 1993).

À la place de cela existe dans l'ouest de l'Afrique Centrale l'idée que le discours active le pouvoir des objets et des substances pour des effets soit bons soit mauvais. Les intentions ne peuvent pas être jugées par le seul discours mais par les effets que le discours et les mots, le ton des sons ou le niveau de bruit ont à la fois sur les choses et les substances qui 
Figure de mascarade Tikar.

Les mondes visibles et invisibles se superposent parfois, et là où cela se passe il y a des portes où des sortes

de formes hétérotopiques visibles

dans des possessions mentales ou des materialisations dans des sanctuaires ou des églises pentecôtistes.

Fig. 6

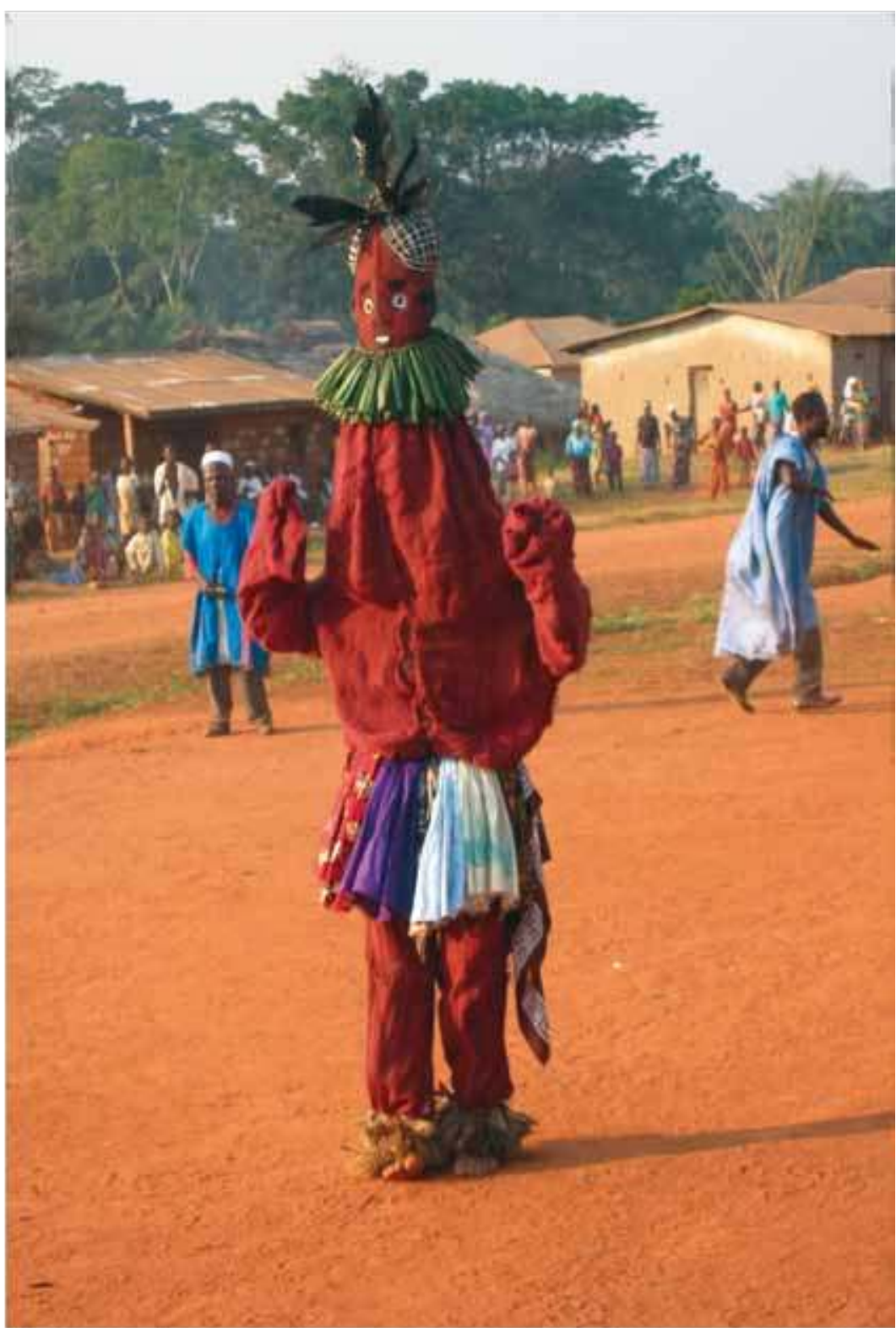

affectent la personne. Ceux-ci incluent les substances du corps, et donc la maladie et la mort. Pendant longtemps, les sectes protestantes extrémistes ont eu du mal à réconcilier leur hostilité de base vis-à-vis des objets matériels tels que les fétiches avec cet usage matérialisé du discours et du son dans de nombreux endroits de l'ouest de l'Afrique Centrale. Les Quakers, par exemple, se fondent sur la spontanéité du discours comme révélation, mais il est également considéré que le discours lui-même a des effets matériels et devrait être sobre et austère. Le pouvoir qu'a le discours de peser sur le monde matériel est profondément ancré dans les offices pentecôtistes, qui incluent des démonstrations d'émotion comme moyen d'attirer l'Esprit Saint. Par le biais de la « possession», le croyant « parle des langues inconnues », dont l'inintelligibilité est une garantie de l'origine divine. Ce qui est rendu confus dans le contexte africain, c'est que le discours lui-même est censé, dans certaines circonstances, provenir des ancêtres et non pas d'une personne vivante. En fait, c'est la raison pour laquelle le discours de colère ou de complainte est puissant, au sens où le discours des ancêtres matérialise la médiation avec les mondes des esprits. Ce discours peut activer ces derniers de sorte qu'ils agissent de façon bienveillante ou malveillante visà-vis des vivants auxquels les mots sont destinés (cf. Dillon 1990). Très souvent, lorsqu'une personne parle fort ou de manière énervée, on entendra quelqu'un dire qu'un « mauvais discours » apporte la malchance, quelle que soit l'intention de ladite personne.

Il n'existe donc pas de différence irréconciliable entre le savoir « africain » concernant la façon dont les mots, les sons et le toucher interagissent avec les mondes invisibles et l'hostilité vis-à-vis des fétiches exercée dans ce scénario par le préjugé des activités missionnaires. Le biais eurocentrique privilégie les mots par rapport aux objets comme un résultat de l'aspect protestant du christianisme. Cette différence est clairement variable, en fonction de la domination de différentes écoles de la pensée chrétienne et de leur impact à travers l'éducation des jeunes. Les Pentecôtistes sont nettement hostiles aux « objets paiens » 
tandis que le Catholicisme s'est montré traditionnellement tolérant. Mais l'impact d'un christianisme plus fondamentaliste dans de nombreux endroits de l'ouest de l'Afrique Centrale, a conduit à faire des mascarades soit une forme de spectacle de danse en groupe par lequel la famille du défunt déploie son prestige, soit des activités secrètes, habituellement nocturnes (Figure 6).

L'idée que le bruit constitue un pont permettant d'établir des connexions suggère que les sons agissent comme une façon de rendre l'invisible visible, comme le principal moyen de médiation pour les mondes matériel et spirituel. Cela peut sembler bizarre, mais le point important n'est ni la séparation conceptuelle du matériel vis-à-vis du spirituel ni le son du visuel. Dans une récente discussion sur les religions africaines, Stephen Ellis et Gerrie ter Haar ont établi une distinction entre les royaumes visibles et invisibles, qu'ils considèrent comme des espaces-temps à part dans les religions africaines (Ellis \& ter Haar 2004). Ils ont développé l'idée que le monde spirituel ou invisible précède le monde matériel et visible dans le temps. Les esprits sont orientés vers le futur et en avance dans le temps par rapport aux vivants. Le monde des esprits n'a en outre aucune contrainte spatiale, c'est-à-dire que les esprits peuvent voyager n'importe où et se trouver à n'importe quel endroit instantanément. Mais les mondes visible et invisible se chevauchent aussi, et là où c'est le cas, il existe des portes où des types d'hérétopies se forment, qui sont matérialisés comme une possession des esprits, ou objectivés dans des autels ou des églises pentecôtistes. Si l'on suit Badiou (2005), cette matérialisation des identités n'est pas seulement principalement rituelle dans sa forme, elle est aussi vécue comme un événement à travers l'acte de spatialisation segmentant et localisant le flux des temporalités.

Mon idée générale est que, en Afrique de l'Ouest non musulmane, de façon très large, c'est le son qui active les objets et les substances, que ce soit dans la forme de la voix, le ton des mots, la musique et plus généralement l'effet percussif. Les objets n'ont pas d'essence fixe, au sens où l'on peut retrouver leur origine, en particulier celle du corps d'un saint ou la compétence d'un auteur particulier. Mais l'idée que certains objets ou sites, une fois activés, peuvent détenir le pouvoir de protéger contre la malveillance et que cela pourrait en outre être renforcé à travers la démonstration de leur efficacité, ne les rend pas irremplaçables. C'est par l'idée que la combinaison des mots, des sons et des objets est efficace pour le refoulement des sentiments d'anxiété que nous pouvons comprendre comment tout cela sert à déplacer les expressions d'horreur vers un autre médium: le film d'horreur.

\section{Du masque au film d'horreur}

Dans le genre occidental du film d'horreur, le monstre est un reflet de la nature du mal qui émerge à travers les actions d'individus. Comme Tobias Wendl l'a souligné, la propagation du film d'horreur en Afrique n'est pas simplement un geste d'adoption, mais davantage une mise en scène et une re-mise en scène de différentes visions de la nature du mal dans des films locaux (Wendl 2001). La sorcellerie est une substance qui, à l'intérieur du corps, peut être activée et prendre différentes formes et différents pouvoirs, par exemple le sorcier, le jujuman, le pasteur du mal ou le voleur 
d'organes corporels pour des rituels occultes. Au Nigéria comme au Ghana, les films dramatiques décrivent les villages et la tradition comme arriérés, pauvres, dangereux et non civilisés. Les craintes vis-à-vis de la sorcellerie, en particulier parmi les jeunes et les urbains, sont dirigées contre les proches et les anciens habitant dans les villages. La différence entre les villages civilisés et la brousse ou la forêt sauvage est transformée pour aboutir à l'idée que les villages sont sauvages, et que la ville représente un espoir de bien-être et de succès. Néanmoins, les villages sont le lieu où tous revendiquent leur origine, le lieu où les corps des défunts doivent être rapportés pour être enterrés, le lieu où l'accès au monde invisible des ancêtres et des esprits sera atteint. D'où la fréquence avec laquelle les villages sont décrits dans les films comme étant envahis par les sorcières et les cannibales, problèmes pris en charge par le héros (un pasteur fondamentaliste, par exemple) qui vient faire le ménage et ramène les habitants à un mode de vie moral. Alors que la ville et, de plus en plus, la vie de la diaspora sont considérées comme incarnant le présent et l'avenir. La diaspora représente aussi le monde de la modernité et de l'innovation qui, incapable de reproduction, est donc stérile. Dans la littérature sur le «bushfalling (impliquant littéralement l'idée que les migrants sont des chasseurs qui vont dans le bush ou l'aspect sauvage de la modernité), les jeunes qui, par le passé, allaient dans la forêt pour chasser de la viande, vont aujourd'hui dans son équivalent urbain moderne pour devenir riche et se procurer les éléments du succès. Mais le «bushfaller » (littéralement la personne qui «tombe » en brousse) ne peut reproduire les conditions du succès sans en rapporter le fruit au village (sous la forme d'envoi de sommes d'argent ou en construisant une maison sur place) (Nyamnjoh 2011).

Les films ont donc le même statut que les masques/mascarades pour la création des conditions permettant d'apporter l'extérieur à l'intérieur. Si les masques représentent l'extérieur, qui est apporté dans le village par les mascarades, c'est à cause de leur caractère hybride africano-européen. Cependant, pour ce qui est des images et des mots, la consommation de films est compatible avec des idéaux chrétiens plus fondamentalistes de l'office religieux, qui privilégie le discours et la prière. Elle est également davantage compatible avec les formes modernisées de discours de sorcellerie liées par certains auteurs à des formes nouvelles d'économie culturelle définies comme la sorcellerie de la richesse par le biais de techniques mystérieuses en elles-mêmes. Ellis \& ter Haar (2004) ont déjà critiqué la tendance à employer des termes comme « occulte », pour faire des généralisations à propos de la nature de la religion africaine. Le problème de ce terme est qu'il implique la connotation occidentale d'un mal abstrait, séparé de ce qui se produit dans la vie quotidienne matérielle. Une définition pratique de la religion africaine suppose un sens plus immanent de l'efficacité matérielle à travers une médiation directe. D'où le fait que, si les mascarades ne sont représentatives de rien, mais sont littéralement considérées comme possédant des moyens importants pour mobiliser les esprits des mondes invisibles, alors il peut en être de même pour les films. Ce ne sont pas de simples films d'horreur dans le sens freudien de la décharge du refoulé: ils constituent plus littéralement un moyen de décrire l'efficacité d'un monde invisible intervenant dans les conditions de la vie matérielle. 


\section{$\&$}

Ma pensée s'articule ici autour de l'idée que la valeur d'irremplaçabilité n'est pas nécessairement due au caractère d'unicité. L'idée d’objets uniques et irremplaçables, habituellement jumelée à une certaine notion d'attachement émotionnel, que nous associons dans des termes psychanalytiques à la discussion de Melanie Klein (1968) sur les objets bons et mauvais ou aux écrits de John Bowbly (1978) sur la théorie de l'attachement, est, me semble-t-il, plus profondément ancrée dans les idées chrétiennes anciennes du pouvoir des reliques et de leur capacité à créer une relation humaine singulière fondée sur les soins et la guérison. J'ajouterais que la « création » du fétiche africain fut le produit de l'anxiété européenne vis-à-vis du pouvoir des reliques, puisqu'il était son contraire. Mais une telle chose était aussi fondée sur le principe inverse selon lequel l'idée de l'irremplaçabilité des reliques (liée au pouvoir du corps comme sacrifice dans le christianisme) a également trouvé son inverse en Afrique; l'idée que l'irremplaçable dépend du remplaçable dépend elle-même du fait d'« intégrer l'extérieur à l'intérieur ». Je pense qu'il nous faut comprendre l'économie des choses remplaçables, ce qui nous permettrait d'identifier le caractère irremplaçable des objets. L'étude de leurs différentes formes au fil du temps devrait nous permettre d'appréhender l'irremplaçable comme métaphysique se matérialisant sous différentes formes.

La rencontre des Européens et des Africains a fait partie du processus par lequel la vitalité de la matière a été diabolisée afin d'ouvrir la voie à la « révolution scientifique » au XvII ${ }^{e}$ siècle. Si la matière est inerte et passive, alors contrairement à ce qui se passe « dans la nature », la matière en elle-même inerte, ne présente pas de danger, sauf en raison des effets de l'intervention humaine. L'idée principale de cet article participe donc de l'idée d'un consensus de plus en plus large sur les pouvoirs d'efficacité des choses ou l'idée d'une matière vivante. Mais alors que ces arguments sont également salutaires d'un point de vue politique, ils leur manque le sens du désir qui demeure implicite dans la notion d'irremplaçabilité. De façon similaire à la peluche de couverture comme compagnon de l'enfance de Pierre Lemonnier, l'idée de " grandir » et mettre à l'écart les « choses » enfantines diabolise le désir de perte de la relique mais justifie également notre sentiment adulte et quotidien de collectionneur.

\section{Traduit de l'anglais par Manuel Benguigui}

\section{NOTES}

Illustration d'ouverture : Prêtre pentecôtiste éloignant le mauvais esprit. La sorcellerie est aussi substance et peut être activée et prendre différentes formes et pouvoirs, par exemple le guérisseur (le jujuman) prêtre maléfique ou voleur d'organes destinés à des rituels occultes. 


\section{RÉFÉRENCES}

Ades, D. 2005 Undercover surrealism: Georges Bataille and documents with contributions by Fiona Bradley. London: Hayward Gallery.

Arnold, K. 1995 Materia medica: A new cabinet of medecine and art. London: Wellcome Institute.

Asad, T. 1993 Genealogies of religion. Baltimore: John Hopkins Press.

Badiou, A. 2005 Being and event. London: Continuum Press

Bowbly, J. 1978 Attachement et perte, vol. 1 : L'attachement. Paris, PUF.

Bennett, J. 2010 Vibrant matter: A political ecology of things. Duke University Press: Durham NC.

Brown, P. 1982 The Cult of the Saints: Its rise and function in latin christianity. Chicago: University of Chicago Press.

Classen, C. 2005 Touch in the museum. In C. Classen (ed.) The Book of Touch. Oxford: Berg: 275-287.

Descola, P. 2010 La Fabrique des images. Paris: Musée du Quai Branly.

Dillon, R. 1990 Ranking and resistance. Stanford: Stanford University Press

Ellis, S., ter Haar, G. 2004 Worlds of Power: Religious thought and political practice in Africa. Oxford: Oxford University Press.

Freud, S. 2002 Civilization and its discontents. London: Penguin.

Geisbusch, J. 2008 Awkward objects: relics, the making of religious meaning and the limits of control in the information age. Londres: University of London (Thèse).

Geary, P. J. 1986 Sacred commodities: the circulation of medieval relics. In A. Appadurai (ed) The Social life of things: commodities in cultural perspective. Cambridge: Cambridge University Press: 169-191.

Geary, P. J. 1990 (1978) Furta Sacra: thefts of relics in the Central Middle Ages. Princeton: Princeton University Press.

Gell, A. 1998 Art and agency: an anthropological theory. Oxford: Clarendon Press.

Graeber, D. 2005 Fetishism and social creativity, or fetishes are gods in process of construction Anthropological Theory 5 (4): 407-438.

Jaucourt, L. de 1765 article « Relique ». In Encyclopédie ou Dictionnaire Raisonné des Sciences, des Arts et des Métiers. Consulté sur internet le 2 février 2007 [portail. atilf. fr/cgi-in/getobject_? a.104: 149./var/ artfla/encyclopedie/textdata/IMAGE//]

Klein, M. 1968 Envie et gratitude. Paris, Gallimard.

Kürzeder, C. 2005 Als die dinge heilig waren: gelebte frömmigkeit im zeitalter des barock. Regensburg: Schnell $\&$ Steiner.

Latour, B. 1993 We have never been Modern, (traduction Catherine Porter). New York, London: Harvester Wheatsheaf.

Latour, B. 2004 Reassembling the social. Oxford: Oxford University Press.

Morris, C. 1972 A critique of popular religion: Guibert of Nogent on the relics of the saints. In G.J. Cuming et D. Baker Popular belief and practice. Cambridge: Cambridge University Press: 55-60.

Nyamnjoh, F. 2012 Cameroonian bushfalling, American Ethnologist 38 (4) : 701-713.

Ogundele, W. 2003 Omoluabi: Ulli Beir, Yoruba society and culture. Bayreuth African Studies 66.

Pietz, W. 1985 The Problem of the Fetish 1, Res $9:$ 5-17.

Pels, P. 1998 The Spirit of matter. On fetish, rarity, fact and fancy. In P. Spyer (ed.) Border fetishisms. London: Routledge: 91-122.

Probst P. 2007 Picturing the past: heritage and photography and the politics of appearance in a Yoruba city. In F. de Jong et M. Rowlands (dir.) Reclaiming heritage. Walnut Creek, California: Left Coast Press.

Recht, R. 2008 Believing and seeing: the art of gohtic cathedrals (traduction Mary Whittall). Chicago: University of Chicago press.

Rowlands, M. 2007 The Sound of Witchcraft: noise as mediation in religious tradition. In: D. Berliner, D. et R. Sarro (ed.) Learning religion Oxford: Berghahn: 191-208.

Wendl, T. 2001 Vision of modernity in Ghana. Mami Wata shrines, photo studios and horror films. Visual Anthropology 14: 269-292. 


\section{RÉSUMÉ}

De la mascarade au film vidéo. Transmettre le diabolique dans le centre de l'Afrique de l'Ouest. Dans cet article, par objet irremplaçable, l'auteur réfère à la possibilité de penser une aire culturelle comme définie par la possession d'un objet significatif qui, d'une certaine manière, lui confère son identité unique dans un contexte global (les combats de taureaux en Espagne ou la cérémonie du thé au Japon). À en juger par leur nombre dans les musées et les collections privées et par les quantités produites pour le marché touristique, l'objet-fétiche qui se présente comme un irréductible stéréotype culturel est le masque, dont le caractère unique d'objet authentiquement « primitif » (africain) renvoie aux rencontres des Européens avec l'Afrique qui sont à l'origine du concept de fétiche. Il est impossible de séparer l'impact des peurs chrétiennes des reliques/fétiches sur les modes africains d'interprétation des relations avec un monde invisible d'esprits pensés comme ayant des effets matériels. La découverte des fétiches sur les côtes d'Afrique de l'Ouest a coïncidé avec la répression du culte des reliques dans l'Europe de la Contre-Réforme.

Au lieu de l'expérience européenne de la relique comme signe de béatification, le fétiche devient le signe d'une médiation avec un monstrueux monde de sorcellerie, de mal et d'horreur - son contraire. Avec les reliques, au sens chrétien médiéval, le lien direct entre le monde invisible et l'objet est l'incarnation physique du Christ et des Apôtres, ainsi que les béatifications ultérieures des saints et les qualités métonymiques du toucher, de la tenue, et du contact avec leurs substances corporelles. Avec l'interprétation du masque comme fétiche vint la vision alternative ou opposée selon laquelle, littéralement, un esprit matériel pouvait envahir l'objet et visiter un monde invisible et potentiellement hostile, et que le contact avec l'objet fétiche corromprait la chair et endommagerait l'âme. Largument final de l'article est qu'avec la montée des christianismes fondamentalistes et des mouvements islamiques à partir des années 1950, les vidéo-drames on repris le rôle de médiation avec le monde invisible à travers le pouvoir des images, plutôt que celui des objets.

\section{ABSTRACT}

From masquerade to video-film: mediating the demonic in West Africa. In this paper, by irreplaceable object the author means the capacity to think of a cultural region as defined by the possession of a significant object image that somehow gives it unique identity in a comparable global setting (bull fighting in Spain or Japanese tea ceremony). In West Africa - witnessed by the sheer number in museum and private collections and the quantities produced for the tourist market -, the irreducible fetish-like object as cultural stereotype is undoubtedly the mask, the uniqueness of which as authentic to primitivism (African primitivism) in turn relates it to European encounters with Africa as the origin of the concept of fetish. It is impossible to separate the impact of the Christian fears of the fetish/relic on African modes of interpreting relations with invisible worlds of spirits that were conceived to have material effects. The discovery of the fetish along the West African coast was coincidental with the repression of relics in counter-reformationist Europe.

Unlike the Christian experience of the relic as a sign of beatification, the fetish became its opposite - a sign of the material mediation to a monstrous world of sorcery / evil and horror. With the relic in the medieval Christian sense the direct link between the invisible world and the object is the physical embodiment of Christ and apostles and subsequent beatifications of saints and the metonymic qualities of touch, handling and contact of their body substances. With the interpretation of the mask as fetish came the alternative or inverted version that literally material spirit could invade the object and visit an invisible and potentially hostile spirit world and contact with the fetish object would corrupt the flesh and damage the soul.

My final argument is that with the rise of more fundamentalist Christianities and Islamic movements from the 1950s onwards, Nigerian and Ghanaian videodramas have taken on the role of mediating with invisible worlds through the power of images rather than objects. 